\title{
Active Release of Microobjects Using a MEMS Microgripper to Overcome Adhesion Forces
}

\author{
Brandon K. Chen*, Student Member, IEEE, Yong Zhang*, Student Member, IEEE, and \\ Yu Sun, Senior Member, IEEE
}

\begin{abstract}
Due to force scaling laws, large adhesion forces at the microscale make rapid accurate release of microobjects a longstanding challenge in pick-place micromanipulation. This paper presents a new microelectromechanical systems (MEMS) microgripper integrated with a plunging mechanism to impact the microobject for it to gain sufficient momentum to overcome adhesion forces. The performance was experimentally quantified through the manipulation of $7.5-10.9-\mu \mathrm{m}$ borosilicate glass spheres in an ambient environment under an optical microscope. Experimental results demonstrate that this microgripper, for the first time, achieves a $100 \%$ successful release rate (based on 200 trials) and a release accuracy of $0.70 \pm 0.46 \mu \mathrm{m}$. Experiments with conductive and nonconductive substrates also confirmed that the release process is not substrate dependent. Theoretical analyses were conducted to understand the release principle. Based on this paper, further scaling down the end structure of this microgripper will possibly provide an effective solution to the manipulation of submicrometer-sized objects.

[2008-0304]
\end{abstract}

Index Terms-Active release, adhesion forces, microelectromechanical systems (MEMS) microgripper, micromanipulation, scaling laws.

\section{INTRODUCTION}

$\mathbf{P}$ HYSICAL pick-place of microobjects promises specificity, precision, and programmed motion, features that make micromanipulation amenable to automation for the construction of microsystems [1]-[4]. For instance, micromanipulation has been used to build a diamond-shaped structure by assembling microspheres into a lattice [1]. Based on a combination of microfabrication and micromanipulation [3], novel photonic crystals were also demonstrated.

Analogous to manipulation in the macroworld, manipulating micrometer-sized objects necessitates gripping devices with end structures comparable in size to objects to be manipulated. Enabled by microelectromechanical systems (MEMS) technologies, many microgripping devices have been reported, including two-fingered devices [5]-[15] and multifingered devices [16]-[22].

Manuscript received December 12, 2008; revised February 27, 2009. First published May 12, 2009; current version published June 3, 2009. This work was supported by the Natural Sciences and Engineering Research Council of Canada through a discovery grant, the Ontario Ministry of Research and Innovation through an Early Researcher Award and a POP grant, the Canada Research Chairs Program, and CMC Microsystems through a microfabrication travel grant. Subject Editor A. J. Ricco.

* Shared first coauthorship. Contributed equally.

The authors are with the Advanced Micro and Nanosystems Laboratory, University of Toronto, Toronto, ON M5S 3G8, Canada (e-mail: brandon.chen@utoronto.ca; yzhang@mie.utoronto.ca; sun@mie.utoronto.ca).

Color versions of one or more of the figures in this paper are available online at http://ieeexplore.iee.org.

Digital Object Identifier 10.1109/JMEMS.2009.2020393
Despite the availability of MEMS gripping tools and the significant progress made in automation techniques for eventually autonomous operation, micromanipulation is still largely skill dependent and entails repeated trial-and-error efforts. Among the challenges, a long-standing difficulty is the release of microobjects from the end effector due to strong adhesion forces at the microscale. Force scaling causes surface forces (i.e., adhesion forces), including the capillary force, the electrostatic force, the and van der Waals force, to dominate volumetric forces (e.g., gravity) [23]. In pursuit of rapid accurate release methods, several strategies have been proposed in the past decade.

These release techniques can be classified into two categories, namely, passive release and active release. Passive release techniques depend on the adhesion forces between the microobject and the substrate to detach the microobject from the end effector. In consideration of adhesional and rolling-resistance factors [24], [25], microspheres were rolled on a Au-coated substrate for both pick and release, causing the fracture of the microsphere-substrate interface and the microsphere-tool interface, respectively. Similarly, it was also demonstrated that ultraviolet cure adhesive was applied on the substrate to enable release [26]. A commonality of passive release techniques includes the dependence on surface properties, being time consuming, and being poor in repeatability.

Differently, active release methods intend to detach the microobject from the end effector without touching the substrate. By applying a voltage between the probe and the substrate [27]-[30], an electric field was created to detach the object from the probe. Nevertheless, this method requires the microobject, the probe, and the substrate all to be conductive. More importantly, the released microobjects landed at random locations on the substrate, resulting in a poor release accuracy.

The second type of active release makes use of mechanical vibration [31], [32]. Requiring a large bandwidth of the manipulator, the vibration-based method takes advantage of inertial effects of both the end effector and the microobject to overcome adhesion forces. The release process has been modeled and simulated to predict the landing radius of the released object [33]; however, the accuracy has not been experimentally quantified. The third type of active release employs vacuum-based tools [34] to create a pressure difference for pick and release. However, miniaturization and accurate control of vacuum-based tools can be difficult, and its use in a vacuum environment can be limited. Finally, micro Peltier coolers were used to form ice droplets instantaneously for pick-place of microobjects [35]-[37]. Thawing of the ice droplets was used to 


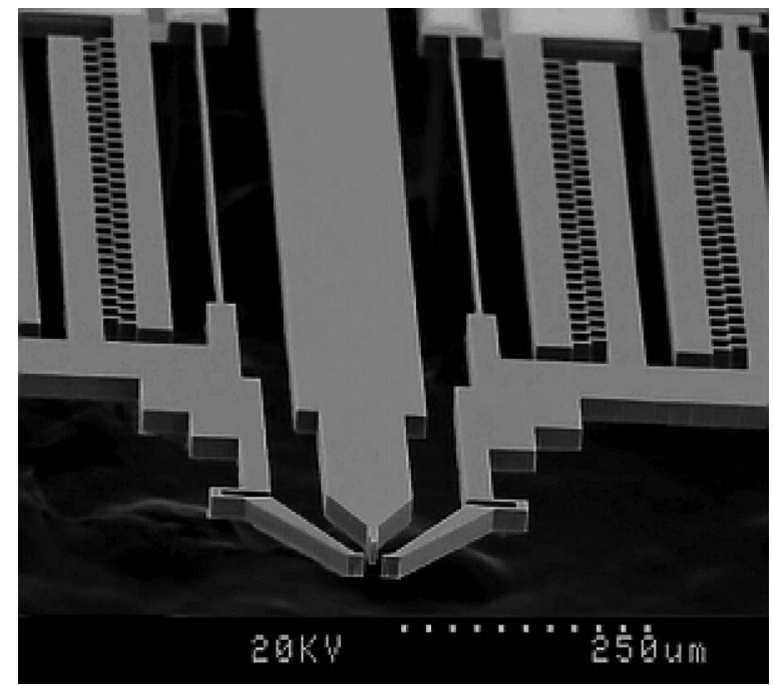

Fig. 1. SEM image of a three-pronged microgripper that is capable of both grasping and active release.

release objects. The freezing-heating approach requires a bulky complex end effector and is limited to micromanipulation in an aqueous environment.

To our best knowledge, no techniques exist for easy, rapid, accurate, and highly repeatable release of microobjects in micromanipulation. In this paper, we present an active release strategy by using a MEMS microgripper that integrates a plunging structure between two gripping arms, as shown in Fig. 1. While the method retains the advantage of double-ended tools for picking up microobjects, the plunger is capable of thrusting a microobject adhered to a gripping arm to a desired destination on a substrate, enabling highly repeatable release with a high accuracy of $0.70 \pm 0.46 \mu \mathrm{m}$.

This paper also presents the theoretical analyses of the micromanipulation process in order to understand the microphysics behind this active release technique. All the preliminary results that this paper describes were obtained under an optical microscope with 7.5-10.9- $\mu \mathrm{m}$ borosilicate microspheres on glass and steel substrates in an ambient environment. No surface treatments were conducted to the microspheres, microgripper, or substrates.

\section{Three-Pronged MicrogripPER}

Fig. 2 shows a schematic of the microgripper. The monolithic device integrates three electrostatic microactuators for driving two normally open gripping arms, as well as a plunger for active release. In this design, electrostatic actuation was chosen over electrothermal actuation because temperature rise of the gripping arms can influence adhesion forces [38] and reduce the consistency of device performance. Furthermore, electrostatic actuation was also chosen for driving the plunger because it exhibits a much higher bandwidth than electrothermal actuators and is able to deliver a much faster speed, representing an important advantage for thrusting off an adhered microobject.

This design is different from existing microgrippers that have either only one actively actuated gripping arm [7]-[9] or two interdependently active gripping arms [5]. Since to which gripping arm a microobject adheres is random, both gripping arms

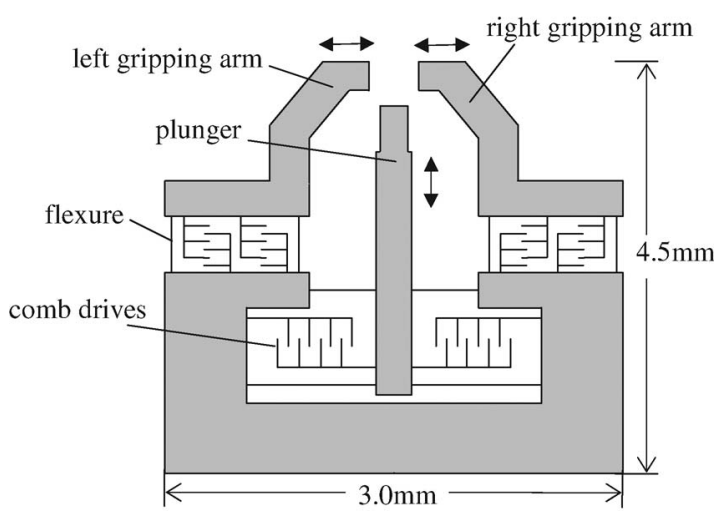

Fig. 2. Microgripper schematic.

TABLE I

Structural Parameters of the MicrogripPers

\begin{tabular}{l|l}
\hline parameter & value \\
\hline device Si layer thickness $(t)$ & $25 \mu \mathrm{m}$ \\
width of gripping arm tips & $6 \mu \mathrm{m}$ \\
width of plunger tip & $8 \mu \mathrm{m}$ \\
initial opening of gripping arms & $17 \mu \mathrm{m}$ \\
gap between comb fingers $(b)$ & $4 \mu \mathrm{m}$ \\
finger pair number of each gripping arm & 280 \\
finger pair number of plunger & 540 \\
width of all flexible beams & $5 \mu \mathrm{m}$ \\
length of flexible beams of gripping arms & $580 \mu \mathrm{m}$ \\
number of flexible beams of each gripping arm & 2 \\
length of flexible beams of plunger & $630 \mu \mathrm{m}$ \\
number of flexible beams of plunger & 4 \\
\hline
\end{tabular}

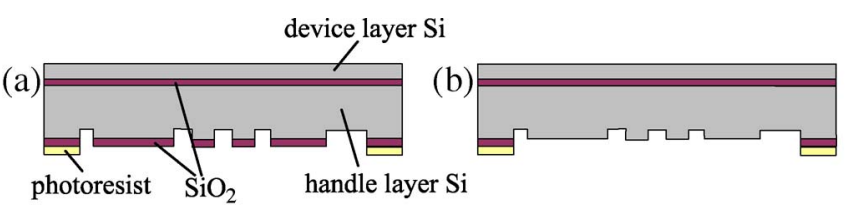

(c)
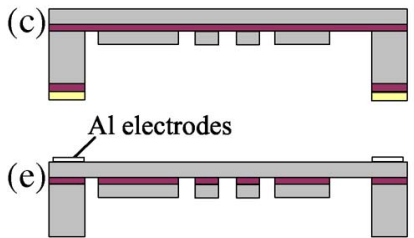

(d)

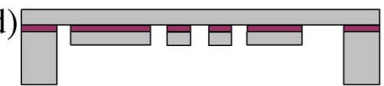

(f)

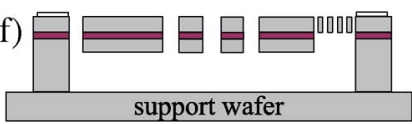

Fig. 3. Microfabrication process.

in our design have an independent actuator for positioning the adhered object to properly align to the plunger for release. The structural parameters of the microgrippers are shown in Table I.

The devices were microfabricated using a modified deep reactive-ion etching (DRIE) on the SOI process [8] with a 25- $\mu \mathrm{m}$-thick device silicon layer, as shown in Fig. 3. Two-step DRIE of the handle layer creates a step difference between the central suspended structure and the device frame, which greatly reduces the risk of device breakage during device operation and handling. Fig. 4 shows the experimentally characterized device performance, as well as fitted lines.

The device also permits one to experimentally estimate the adhesion forces between the gripping arms and a grasped microsphere. After the microsphere is gently but securely grasped, the actuation voltages for the gripping arms are released in 


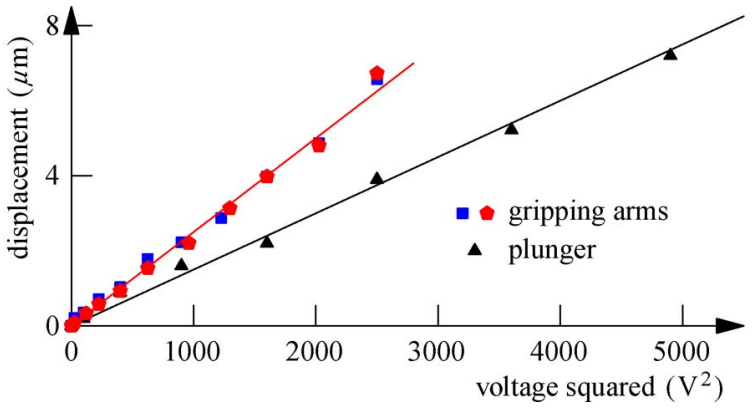

Fig. 4. Characterized microactuator performance.

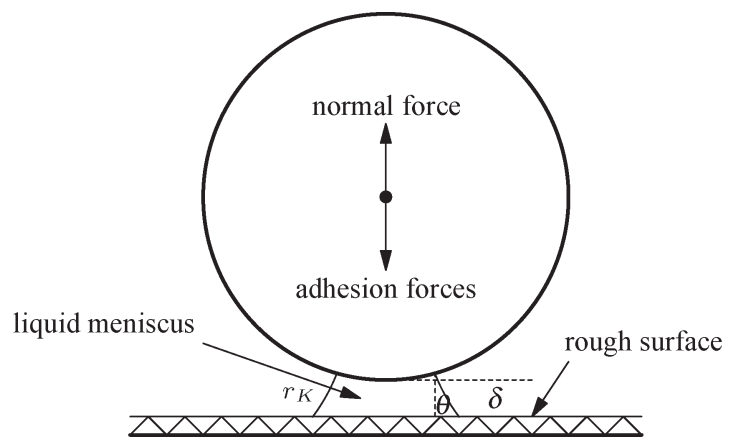

Fig. 5. Adhesion forces acting on a microsphere on a rough surface.

a continuous and synchronous manner until the voltage $V_{2}$ at which the gripping arms are opened is obtained. The adhesion forces can then be estimated as

$$
F=\frac{1}{2} \frac{N \epsilon t}{b}\left(V_{1}^{2}-V_{2}^{2}\right)
$$

where $\epsilon$ is the permittivity of air, $N$ is the number of comb finger pairs, $t$ is the comb finger thickness, $b$ is the gap between opposing comb fingers, and $V_{1}$ is the voltage applied to both gripping arms for creating a gap of the size of the microobject. Note that when the initial grasping force applied to the microobject is increased, the actuation voltage $V_{2}$ required for opening the gripping arms becomes smaller, resulting in a larger estimate of the adhesion forces. Despite the initial grasping force variations, as well as microfabrication-induced imperfections, the adhesion forces obtained through this actuation force estimation proved useful for understanding purposes.

\section{Force ANALYSis}

Adhesion forces in an ambient environment include three types of attractive forces, namely, the van der Waals force, the electrostatic force, and the capillary force, all of which depend on the separation distance $\delta$ between a microsphere and a flat surface that it adheres to. Fig. 5 shows a microsphere adhered to a flat surface with surface roughness exaggerated.

Van der Waals forces are caused by the instantaneous polarization of atoms and molecules due to quantum-mechanical effects. The van der Waals force between a microsphere and a flat surface is [39]

$$
F_{\mathrm{vdw}}=\left(\frac{\delta}{\delta+r / 2}\right)^{2}\left(\frac{H d}{16 \pi \delta^{2}}+\frac{H \rho^{2}}{8 \pi \delta^{3}}\right)
$$

where $r$ is the roughness of the flat surface, $H$ is the Lifshitz-van der Waals constant that ranges from $0.6 \mathrm{eV}$ for polymers to $9.0 \mathrm{eV}$ for metals, $d$ is the microsphere diameter, and $\rho$ is the radius of the adhesion surface area.

To estimate the van der Waals force between a $10-\mu \mathrm{m}$ borosilicate microsphere and the sidewall of a gripping arm, $\delta$ is assumed to be $0.35 \mathrm{~nm}$ [38], $\rho$ is assumed to be $0.65 \%$ of the radius of the microsphere [38], $H$ is assumed to be $7.5 \mathrm{eV}$ [38], and $r$ is assumed to be $100 \mathrm{~nm}$. Thus, the van der Waals force is calculated to be $1.51 \times 10^{-4} \mu \mathrm{N}$.

The electrostatic force for microspheres that are smaller than $100 \mu \mathrm{m}$ is predominantly the electrostatic double-layer force, which is [40]

$$
F_{\text {elec }}=\frac{\pi \epsilon d U^{2}}{2 \delta}
$$

where $\epsilon$ is the permittivity of air and $U$ is the voltage difference between the microsphere and the flat surface. When $U$ is assumed to be $0.40 \mathrm{~V}$ [38], the electrostatic force between a $10-\mu \mathrm{m}$ microsphere and the sidewall of a gripping arm is calculated to be $6.36 \times 10^{-2} \mu \mathrm{N}$.

The third type of attractive force is the capillary force, which is composed of the capillary pressure force and the surface tension force [41], [42]. The capillary pressure force dominates the surface tension force for microspheres that are larger than $1 \mu \mathrm{m}$ [41]. For the microsphere-plane model, the capillary force is [43], [44]

$$
F_{\text {cap }}=\frac{2 \pi d \gamma \cos \theta}{1+\delta /\left(2 r_{K} \cos \theta-\delta\right)}
$$

where $\gamma$ is the liquid surface tension, which is $0.073 \mathrm{~N} \cdot \mathrm{m}^{-1}$ for water at $22{ }^{\circ} \mathrm{C}, \theta$ is the contact angle of the meniscus with the microsphere, and $r_{K}$ is the Kelvin radius, which is defined as the mean radius of the curvature of the liquid-vapor interface.

For estimating the capillary force exerted on a $10-\mu \mathrm{m} \mathrm{mi-}$ crosphere by a water meniscus at room temperature, $\theta$ is assumed to be $10^{\circ}, \delta$ is still assumed to be $0.35 \mathrm{~nm}$ as for the calculation of the van der Waals force, and $r_{K}$ is assumed to be $1 \mathrm{~nm}$. The capillary force is calculated to be $3.71 \mu \mathrm{N}$.

For comparison purposes, the gravity of the $10-\mu \mathrm{m} \mathrm{mi-}$ crosphere is calculated to be $1.31 \times 10^{-5} \mu \mathrm{N}$, using the density of borosilicate glass that is $2.55 \mathrm{~g} / \mathrm{cm}^{3}$. In summary, the pecking order is

$$
F_{\text {cap }} \gg F_{\text {elec }} \gg F_{\text {vdw }} \gg F_{\text {grav }} .
$$

It can be seen that the van der Waals force is the smallest among the three attractive forces. The van der Waals force heavily depends on the roughness of the surface. Since devices were formed through DRIE, which produces scallop structures on the sidewalls of the gripping arms, the rough surface makes the van der Waals force negligible. The electrostatic force depends on voltage differences, which are difficult to accurately estimate when the microsphere is nonconductive. Unlike the van der Waals force and electrostatic force, neither of which requires physical contact, the capillary force in the air results from a phenomenon called capillary condensation [40]. Liquid from the vapor phase condenses between sufficiently close asperities and forms menisci that cause the capillary force. Thus, 
(a)

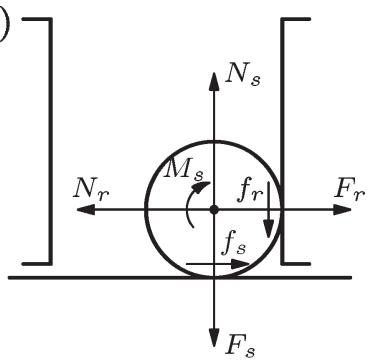

(c)

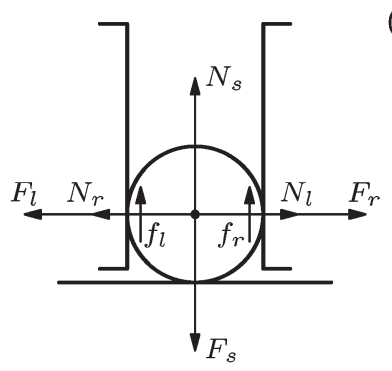

(e)

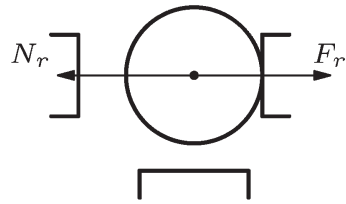

(b)

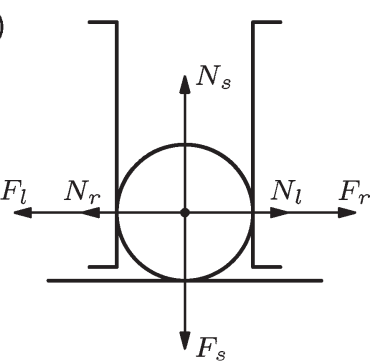

(d)

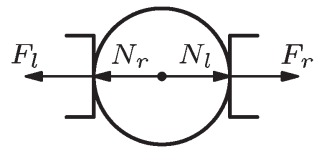

(f)

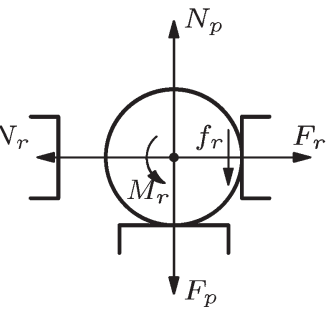

Fig. 6. Analysis of forces during grasping and active release.

there exists a working range, beyond which the capillary force, as well as the liquid menisci, disappears.

The schematic diagrams in Fig. 6 show the forces exerted on a microsphere by the gripping arms and/or the substrate during grasping and release. Fig. 6(a)-(c) is drawn from the side view, and Fig. 6(d)-(f) is from the top view. Fig. 6(a) shows that the microgripper approaches the microsphere and uses the gripping arm to laterally push it in order to break the adhesion bond between the microsphere and the substrate. $F_{s}$ is the adhesion force, and $N_{s}$ is the normal force from the substrate. $N_{r}$ is the laterally pushing force applied by the right gripping arm, and $F_{r}$ is the adhesion force from the gripping arm in the normal direction. Upon the application of $N_{r}$, the stress distribution in the contact area between the microsphere and the substrate becomes nonuniform, which creates a rolling-resistance moment $M_{s}$ [45], [46]. Other than the adhesion forces $F_{s}$ and $F_{r}$ that are normal to the flat surfaces, $f_{s}$ and $f_{r}$ are additional capillary forces from the substrate and the gripping arm, respectively. $f_{s}\left(f_{r}\right)$ resists the relative motion between the microsphere and the substrate (gripping arm) through the menisci. In this situation, the total capillary forces from the substrate and gripping arm are not perpendicular to the flat surfaces.

After the microsphere is moved laterally from its original position, the two gripping arms close and grasp it, as shown in Fig. 6(b). The normal and adhesion forces $N_{l}$ and $F_{l}$ are from the left gripping arm. Similarly, $N_{r}$ and $F_{r}$ are from the right gripping arm. Aside from $F_{l}$ and $F_{r}$, there can also be additional capillary forces parallel to the substrate surface and gripping arm surface, although they are not shown in the diagram for clarity.

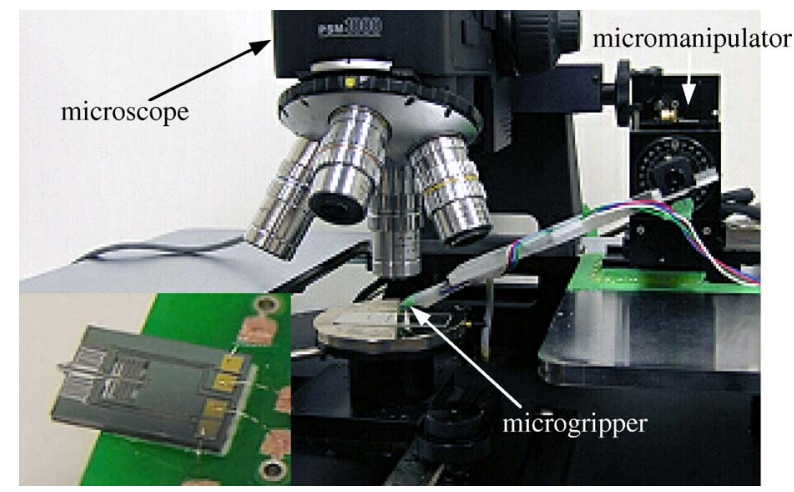

Fig. 7. Experimental setup for micrograsping and active release tests. The inset shows a wire-bonded microgripper.

The microgripper is then raised, as shown in Fig. 6(c) to lift up the microsphere. The additional capillary forces from the gripping arms, $f_{l}$ and $f_{r}$, overcome the adhesion forces from the substrate, $F_{s}$, which decreases gradually as a function of the distance between the microsphere and the substrate.

When the microsphere is up in the air [Fig. 6(d)], the adhesion forces from the substrate become negligible. Upon reaching a desired destination, the gripping arms are opened, during which all of the adhesion and normal forces from the gripping arms decrease. Consequently, the microsphere separates from one gripping arm and keeps adhering to the other gripping arm by adhesion forces, as shown in Fig. 6(e).

For release, the gripping arm with the adhered microsphere is properly positioned relative to the plunger, as shown in Fig. 6(f). The plunger is then controlled to move forward to thrust out and collide with the microsphere. The microsphere eventually escapes from the adhesion forces from the gripping arm by its own inertia and lands on the substrate. In Fig. 6(f), $N_{p}$ is the pushing force applied by the plunger, $F_{p}$ is the adhesion force from the plunger, and $M_{r}$ and $f_{r}$ are the rollingresistance moment and additional capillary force from the gripping arm, respectively.

\section{EXPERIMENTAL RESUlTS AND DisCUSSION}

The experimental setup (Fig. 7) consists of an optical microscope (Motic PSM-1000) with a CMOS camera (Sony XCD710). A custom-made circuit board with a wire-bonded microgripper was mounted on a 3-DOF micromanipulator (Sutter MP-285) at a tilting angle of $25^{\circ}$.

Borosilicate glass microspheres (diameters: 7.5-10.9 $\mu \mathrm{m}$ ) were manipulated at room temperature of $22{ }^{\circ} \mathrm{C}$ with relative humidity of $50 \pm 5 \%$. A droplet of microspheres in isopropanol was micropipetted onto the substrate and let dry in air. The surface tension of isopropanol $(0.021 \mathrm{~N} / \mathrm{m}$ at room temperature $)$ is smaller than that of water. However, due to the volatility of isopropanol and because the microspheres were let dry in air for a prolonged period, water was assumed to constitute most of the liquid menisci between the microspheres and the substrate. Therefore, the surface tension of water was used in (4) in Section III for estimating the capillary force.

Two types of substrates, wipe cleaned with isopropanol and let dry in air, were used in the experiments, including an 
(a)

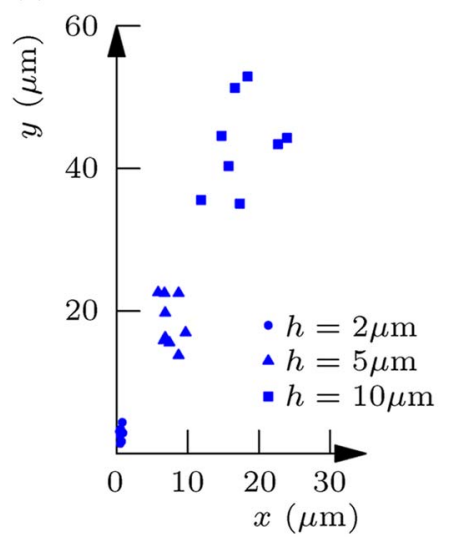

(b)

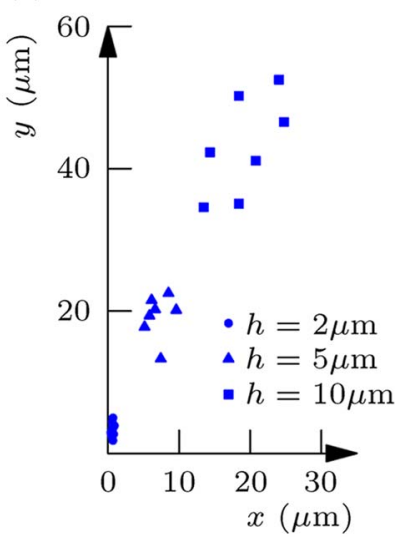

Fig. 8. Landing positions of microspheres. $h$ is the height of the gripping arms from above the substrate. (a) Glass substrate. (b) Steel substrate.

electrically conductive substrate (steel) and a nonconductive substrate (glass). These two substrates were expected to exert different electrostatic forces and van der Waals forces on the microsphere while it is traveling in air during release, which might affect the release accuracy.

\section{A. Repeatability of Active Release}

After the gripping arms opened, the microsphere randomly adhered to a gripping arm in all cases. The overall adhesion forces between the gripping arms and the microsphere were estimated to be $3.6-5.8 \mu \mathrm{N}$ by measuring the actuation voltages required to open the gripping arms after a gentle yet secured grasping of a microsphere, as described in (1) in Section II.

For successful release, the microsphere must gain a sufficient amount of momentum from the collision with the plunger in order to overcome the adhesion forces. The speed of the plunger can be varied by controlling the rising time of the actuation voltage. When a sharp increase in actuation voltage was applied to the plunger (e.g., from 0 to $50 \mathrm{~V}$ within $0.1 \mathrm{~s}$ ), release of the microsphere was guaranteed (i.e., $100 \%$ success rate, $n=$ 200). A high plunging speed alleviates careful sample preparation requirements (e.g., baking) or environmental control requirements (e.g., humidity). Quantification using high-speed videography (13000 frames/s) revealed that a plunging speed of $65.24 \mathrm{~mm} / \mathrm{s}$ produced a microsphere speed of $105.01 \mathrm{~mm} / \mathrm{s}$. High-speed videography also demonstrated that a microsphere was separated from the plunger upon impact.

\section{B. Quantification of Release Performance}

To quantitatively characterize the release performance, single microspheres were repeatedly picked and released from different heights $(2-30 \mu \mathrm{m})$ above the substrate. Fig. 8(a) shows representative data of landing positions on a glass substrate. The results show a fairly linear and predictable relationship between landing positions and heights from the substrate, indicating that forces including the van der Waals forces and the electrostatic forces from both the substrate and the microgripper, as well as the gravitational force, do not have a significant effect on the high-speed microsphere that travels a short distance in air.

(a)

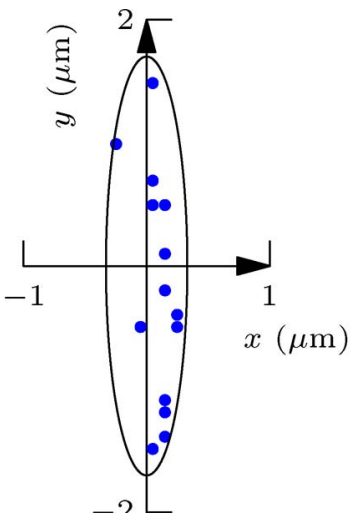

(b)

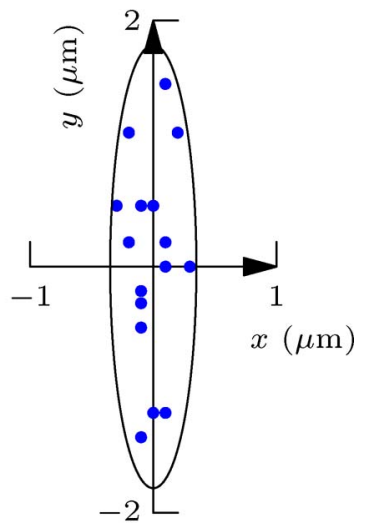

(c)

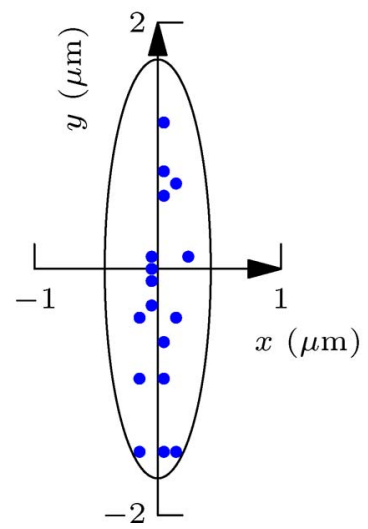

(d)

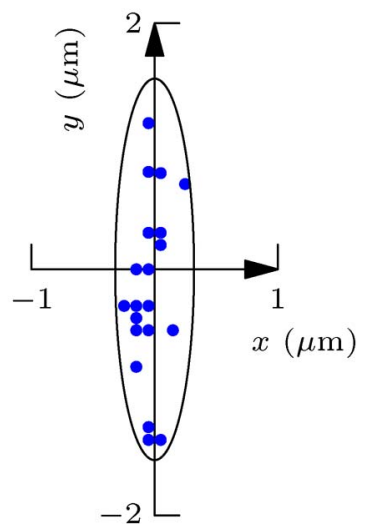

Fig. 9. Landing positions of microspheres when the gripping arms were placed $2 \mu \mathrm{m}$ above the substrate. (a) and (b) Glass substrate. (c) and (d) Steel substrate. (a) and (c) Microspheres adhered to the left gripping arm. (b) and (d) Microspheres adhered to the right gripping arm.

Fig. 8(a) also shows that the precision of landing is inversely proportional to the height from the substrate. When the height was over $20 \mu \mathrm{m}$, random landing locations were observed, which should be partly due to the more pronounced airflow effect. To investigate the influence of substrate differences on release performance, experiments were also repeated using a steel substrate. Compared to the data in Fig. 8(a), the results shown in Fig. 8(b) confirm that the active release approach does not have observable substrate dependence.

As mentioned earlier, adherence of the microsphere to which gripping arm is random. Fig. 8(a) and (b) show the experimental data collected when the microspheres adhered to the right gripping arm. Similar data were captured but not shown for microspheres that adhered to the left gripping arm.

Given the aforementioned findings, the release height was set to $2 \mu \mathrm{m}$ above the substrate for quantifying the release accuracy. The small distance of $2 \mu \mathrm{m}$ from the substrate reduces the distance/time that the microsphere travels in air, making the landing location less sensitive to environmental disturbances. Fig. 9 shows the recorded landing positions of the microsphere, proving an accuracy of $0.70 \pm 0.46 \mu \mathrm{m}$. A summary of the characterized release accuracy is given in Table II. The $0.55-\mu \mathrm{m}$ standard deviation of landing positions can be due to either of the following: 1) slight variations of initially adhering lateral and/or vertical positions of the microsphere on the gripping arm or 2) imperfect control of the microgripper height above the substrate. 
TABLE II

SUMMARY OF RELEASE ACCURACY

\begin{tabular}{c|c|c}
\hline & $\begin{array}{c}\text { glass substrate } \\
\text { release accuracy }\end{array}$ & $\begin{array}{c}\text { steel substrate } \\
\text { release accuracy }\end{array}$ \\
\hline microspheres adhered & $0.70 \pm 0.46 \mu \mathrm{m}$ & $0.67 \pm 0.55 \mu \mathrm{m}$ \\
to left arm & $(n=18)$ & $(n=18)$ \\
\hline $\begin{array}{c}\text { microspheres adhered } \\
\text { to right arm }\end{array}$ & $0.64 \pm 0.46 \mu \mathrm{m}$ & $0.67 \pm 0.55 \mu \mathrm{m}$ \\
$(n=18)$ & $(n=20)$ \\
\hline
\end{tabular}
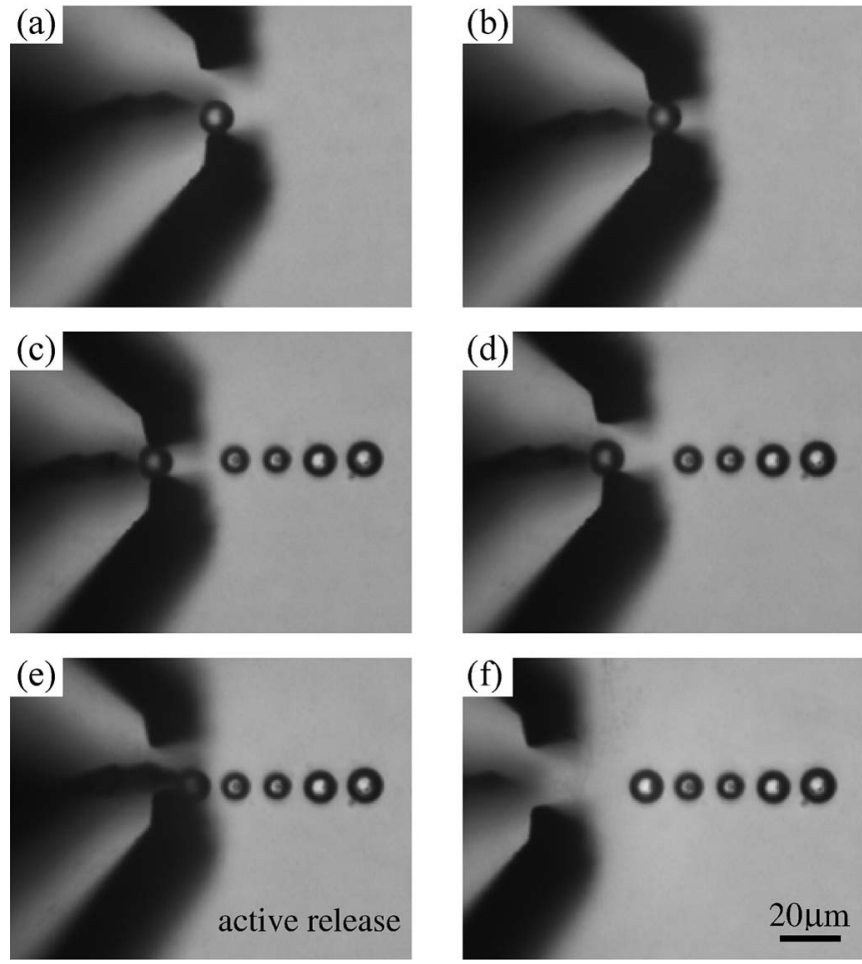

Fig. 10. Pick-place to align microspheres $(7.5-10.9 \mu \mathrm{m})$. Note that the microgripper was tilted by $25^{\circ}$. (a) Microgripper approaches a microsphere and uses one gripping arm to laterally push it to break the initial adhesion bond between the microsphere and the substrate. (b) Two gripping arms are closed, grasping the microsphere and lifting it up. (c) Microsphere is transported to the target area where some microspheres have already been aligned. (d) Gripping arms are opened, and the gripping arm that the microsphere adheres to positions the microsphere properly to the right position in relation to the plunger. (e) Plunger thrusts out the microsphere that lands accurately on the substrate.

(f) Microgripper retracts to repeat the pick-place process.

Other than a high accuracy, the active release technique enables easy fast pick-place operation in micromanipulation. Fig. 10 shows the result of a series of pick and release of microspheres. While grasping was manually conducted, which is skill dependent, positioning the microsphere properly for plunging was rapid and took less than $1 \mathrm{~s}$ with the use of the calibration results shown in Fig. 4. The actual release takes $0.17 \mathrm{~ms}$ according to high-speed videography.

\section{Understanding the Curved Trajectory}

Interestingly, it can be seen from Fig. 8 that the microspheres all landed to the right/left side of the plunger (the plunger was along the $y$-axis), depending on which gripping arm that they adhered to. High-speed imaging verified that the flying path of the microsphere was indeed curved. The images shown in Fig. 11 were taken through high-speed videography when

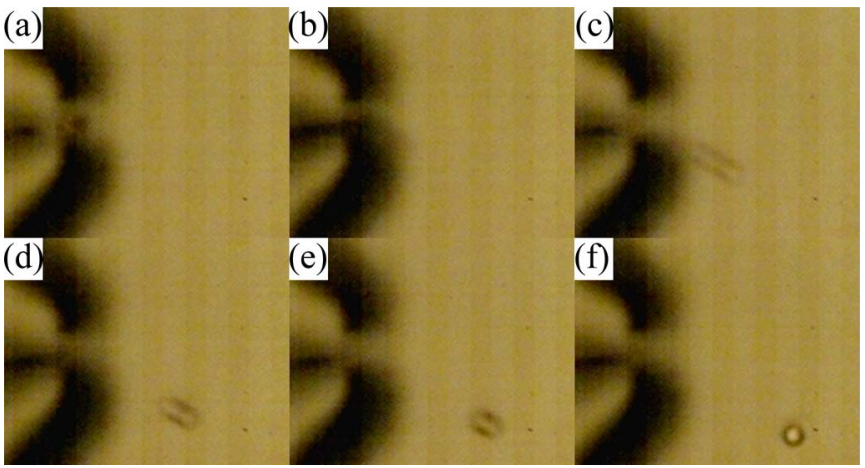

Fig. 11. High-speed videography (13000 frames/s) quantifying microsphere trajectories upon release from a height of $20 \mu \mathrm{m}$ above the substrate.

(a)

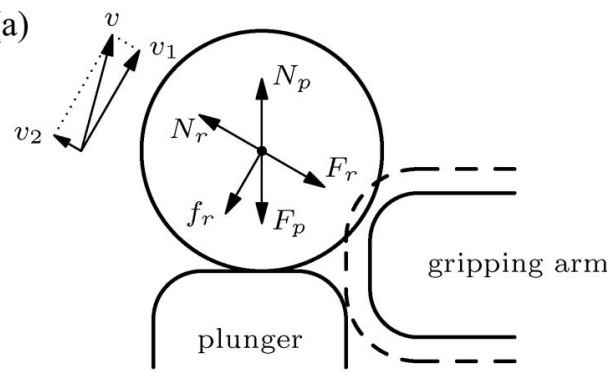

(b)

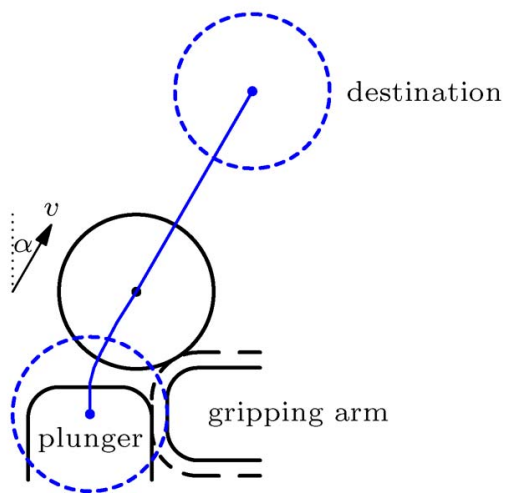

Fig. 12. Microsphere reveals a curved trajectory during active release. (a) Plunger thrusts the microsphere that reaches the roundish corner of the gripping arm. (b) Microsphere escapes from the effective range of the adhesion forces. The trajectory is drawn under the assumption that there are no disturbances when the microsphere is in the air.

the gripping arms were $20 \mu \mathrm{m}$ above the substrate before the release of the microsphere.

According to the brief force analysis in Section III, the van der Waals force and electrostatic force decrease with increased distances between the microsphere and gripping arm. Additionally, the capillary force vanishes beyond a certain distance. Thus, it is assumed that the gripping arm has an adhesion-force effective region around it, as indicated by the dashed lines in Fig. 12.

During release, the plunger first impacts the microsphere along the sidewall of the gripping arm at a high speed, as shown in Fig. 12(a), where the dotted lines represent the adhesionforce effective region. When the traveling microsphere approaches the gripping-arm corner, which was rounded by DRIE, the adhesion forces create a radial acceleration toward the corner, which curves its travel direction. While the microsphere 
is within the adhesion-force effective region, there exists resistance $f_{r}$ (additional capillary force) in the tangential direction caused by menisci. Eventually, the microsphere leaves the gripping-arm tip and, hence, the adhesion-force effective region. It then travels straightly and lands on the substrate, as shown in Fig. 12(b).

\section{CONClusion}

This paper presented a new MEMS microgripper that integrated both gripping and release mechanisms. The microgripper was applied to the grasping and active release of microspheres. The plunger provided the microsphere with sufficient momentum to overcome adhesion forces, resulting in highly repeatable release (100\% of 200 trials) and a release accuracy of $0.70 \pm 0.46 \mu \mathrm{m}$. The tested borosilicate microspheres varied from 7.5 to $10.9 \mu \mathrm{m}$ in size. Within this size range, release accuracy was found independent of microsphere sizes. Release performance was also found independent of electrical conductivity of substrates (steel and glass). Considering the structural dimensions of the present device (e.g., thickness of gripping arms and plunger: $25 \mu \mathrm{m}$, and initial gripping arm opening: $17 \mu \mathrm{m}$ ), we speculate that the reported release accuracy should be consistent for microspheres ranging from a few micrometers up to $17 \mu \mathrm{m}$. This paper revealed that the most important operating parameters are plunging speed and the height from the substrate. The highly controllable active release capability represents an important progress for reliable pick-place micromanipulation. By virtue of its grasping and rapid accurate release capabilities, the MEMS microgripper will enable fully automated micromanipulation.

\section{REFERENCES}

[1] F. García-Santamaría, H. T. Miyazaki, A. Urquía, M. Ibisate, M. Belmonte, N. Shinya, F. Meseguer, and C. López, "Nanorobotic manipulation of microspheres for on-chip diamond architectures," $A d v$. Mater, vol. 14, no. 16, pp. 1144-1147, Aug. 2002.

[2] K. Aoki, H. T. Miyazaki, H. Hirayama, K. Inoshita, T. Baba, N. Shinya, and Y. Aoyagi, "Three-dimensional photonic crystals for optical wavelengths assembled by micromanipulation," Appl. Phys. Lett., vol. 81, no. 17 , pp. 3122-3124, Oct. 2002.

[3] K. Aoki, H. T. Miyazaki, H. Hirayama, K. Inoshita, T. Baba, K. Sakoda, N. Shinya, and Y. Aoyagi, "Microassembly of semiconductor threedimensional photonic crystals," Nat. Mater, vol. 2, no. 2, pp. 117-121, Feb. 2003.

[4] A. Tafazzoli, C.-M. Cheng, C. Pawashe, E. K. Sabo, L. Trofin, M. Sitti, and P. R. LeDuc, "Subfeature patterning of organic and inorganic materials using robotic assembly," J. Mater. Res., vol. 22, no. 6, pp. 1601-1608, Jun. 2007.

[5] N. Chronis and L. P. Lee, "Electrothermally activated SU-8 microgripper for single cell manipulation in solution," J. Microelectromech. Syst., vol. 14, no. 4, pp. 857-863, Aug. 2005.

[6] D. H. Kim, M. G. Lee, B. Kim, and Y. Sun, "A superelastic alloy microgripper with embedded electromagnetic actuators and piezoelectric force sensors: A numerical and experimental study," Smart Mater. Struct., vol. 14 , no. 6 , pp. 1265-1272, Dec. 2005.

[7] F. Beyeler, A. Neild, S. Oberti, D. J. Bell, Y. Sun, J. Dual, and B. J. Nelson, "Monolithically fabricated microgripper with integrated force sensor for manipulating microobjects and biological cells aligned in an ultrasonic field," J. Microelectromech. Syst., vol. 16, no. 1, pp. 7-15, Feb. 2007

[8] K. Kim, X. Liu, Y. Zhang, J. Cheng, X. Y. Wu, and Y. Sun, "Elastic and viscoelastic characterization of microcapsules for drug delivery using a force-feedback MEMS microgripper," Biomed. Microdevices, vol. 11, no. 2, pp. 421-427, Apr. 2009.
[9] C. Yamahata, D. Collard, B. Legrand, T. Takekawa, M. Kumemura, G. Hashiguchi, and H. Fujita, "Silicon nanotweezers with subnanometer resolution for the micromanipulation of biomolecules," J. Microelectromech. Syst., vol. 17, no. 3, pp. 623-631, Jun. 2008.

[10] G. K. Lau, J. F. L. Goosen, F. van Keulen, T. C. Duc, and P. M. Sarro, "Polymeric thermal microactuator with embedded silicon skeleton: Part I-Design and analysis," J. Microelectromech. Syst., vol. 17 , no. 4 , pp. 809-822, Aug. 2008.

[11] T. C. Duc, G. K. Lau, and P. M. Sarro, "Polymeric thermal microactuator with embedded silicon skeleton: Part II-Fabrication, characterization, and application for 2-DOF microgripper," J. Microelectromech. Syst., vol. 17, no. 4, pp. 823-831, Aug. 2008.

[12] O. Sardan, V. Eichhorn, D. H. Petersen, S. Fatikow, O. Sigmund, and P. Bøggild, "Rapid prototyping of nanotube-based devices using topologyoptimized microgrippers," Nanotechnology, vol. 19, no. 49, p. 495503 , Dec. 2008.

[13] Y. Choi, J. Ross, B. Wester, and M. G. Allen, "Mechanically driven microtweezers with integrated microelectrodes," J. Micromech. Microeng., vol. 18 , no. 6, p. 065004 , Jun. 2008.

[14] T. C. Duc, G.-K. Lau, J. F. Creemer, and P. M. Sarro, "Electrothermal microgripper with large jaw displacement and integrated force sensors," J. Microelectromech. Syst., vol. 17, no. 6, pp. 1546-1555, Dec. 2008.

[15] K. N. Andersen, D. H. Petersen, K. Carlson, K. Mølhave, O. Sardan, A. Horsewell, V. Eichhorn, S. Fatikow, and P. Bøggild, "Multimodal electrothermal silicon microgrippers for nanotube manipulation," IEEE Trans. Nanotechnol., vol. 8, no. 1, pp. 76-85, Jan. 2009.

[16] H.-Y. Chan and W. J. Li, "A thermally actuated polymer micro robotic gripper for manipulation of biological cells," in Proc. IEEE Int. Conf. Robot. Autom., Taipei, Taiwan, Sep. 2003, pp. 288-293.

[17] J. K. Luo, J. H. He, Y. Q. Fu, A. J. Flewitt, S. M. Spearing, N. A. Fleck, and W. I. Milne, "Fabrication and characterization of diamond-like carbon/Ni bimorph normally closed microcages," J. Micromech. Microeng., vol. 15, no. 8, pp. 1406-1413, Aug. 2005.

[18] Y.-W. Lu and C.-J. Kim, "Microhand for biological applications," Appl. Phys. Lett., vol. 89, no. 16, p. 164 101, Oct. 2006.

[19] J. Ok, Y.-W. Lu, and C.-J. Kim, "Pneumatically driven microcage for microbe manipulation in a biological liquid environment," J. Microelectromech. Syst., vol. 15, no. 6, pp. 1499-1505, Dec. 2006.

[20] S. Krishnan and L. Saggere, "A multi-fingered micromechanism for coordinated micro/nano manipulation," J. Micromech. Microeng., vol. 17, no. 3, pp. 576-585, Mar. 2007.

[21] J. S. Randhawa, T. G. Leong, N. Bassik, B. R. Benson, M. T. Jochmans, and D. H. Gracias, "Pick-and-place using chemically actuated microgrippers," J. Amer. Chem. Soc., vol. 130, no. 51, pp. 17 238-17239, 2008.

[22] T. G. Leong, C. L. Randall, B. R. Benson, N. Bassik, G. M. Stern, and D. H. Gracias, "Tetherless thermobiochemically actuated microgrippers," Proc. Nat. Acad. Sci. U.S.A., vol. 106, no. 3, pp. 703-708, Jan. 2009.

[23] R. S. Fearing, "Survey of sticking effects for micro-parts," in Proc. IEEE/RSJ Int. Conf. Intell. Robots Syst., Pittsburgh, PA, Aug. 1995, pp. 212-217.

[24] H. T. Miyazaki, Y. Tomizawa, S. Saito, T. Sato, and N. Shinya, "Adhesion of micrometer-size polymer particles under a scanning electron microscope," J. Appl. Phys., vol. 88, no. 6, pp. 3330-3340, Sep. 2000.

[25] S. Saito, H. T. Miyazaki, T. Sato, and K. Takahashi, "Kinematics of mechanical and adhesional micromanipulation under a scanning electron microscope," J. Appl. Phys., vol. 92, no. 9, pp. 5140-5149, Nov. 2002.

[26] O. Fuchiwaki, A. Ito, D. Misaki, and H. Aoyama, "Multi-axial micromanipulation organized by versatile micro robots and micro tweezers," in Proc. IEEE Int. Conf. Robot. Autom., Pasadena, CA, May 2008, pp. 893-898.

[27] K. Takahashi, H. Kajihara, M. Urago, S. Saito, Y. Mochimaru, and T. Onzawa, "Voltage required to detach an adhered particle by Coulomb interaction for micromanipulation," J. Appl. Phys., vol. 90, no. 1, pp. 432437, Jul. 2001.

[28] S. Saito, H. Himeno, and K. Takahashi, "Electrostatic detachment of an adhering particle from a micromanipulated probe," J. Appl. Phys., vol. 93, no. 4, pp. 2219-2224, Feb. 2003.

[29] S. Saito, H. Himeno, K. Takahashi, and M. Urago, "Kinetic control of a particle by voltage sequence for a nonimpact electrostatic micromanipulation,” Appl. Phys. Lett., vol. 83, no. 10, pp. 2076-2078, Sep. 2003.

[30] S. Saito and M. Sonoda, "Non-impact deposition for electrostatic micromanipulation of a conductive particle by a single probe," J. Micromech. Microeng., vol. 18, no. 10, p. 107 001, Oct. 2008.

[31] D. S. Haliyo, S. Régnier, and J.-C. Guinot, "[mü]MAD, the adhesion based dynamic micro-manipulator," Eur. J. Mech. A, Solids, vol. 22, no. 6, pp. 903-916, Nov./Dec. 2003. 
[32] D. S. Haliyo, Y. Rollot, and S. Régnier, "Manipulation of micro objects using adhesion forces and dynamical effects," in Proc. IEEE Int. Conf. Robot. Autom., Washington DC, May 2002, pp. 1949-1954.

[33] Y. Fang and X. Tan, "A dynamic JKR model with application to vibration release in micromanipulation," in Proc. IEEE/RSJ Int. Conf. Intell. Robots Syst., Beijing, China, Oct. 2006, pp. 1341-1345.

[34] W. Zesch, M. Brunner, and A. Weber, "Vacuum tool for handling microobjects with a nanorobot," in Proc. IEEE Int. Conf. Robot. Autom., Albuquerque, NM, Apr. 1997, pp. 1761-1766.

[35] J. Liu, Y.-X. Zhou, and T.-H. Yu, "Freeze tweezer to manipulate mini/micro objects," J. Micromech. Microeng., vol. 14, no. 2, pp. 269 276, Feb. 2004.

[36] Y. Yang, J. Liu, and Y.-X. Zhou, "A convective cooling enabled freeze tweezer for manipulating micro-scale objects," J. Micromech. Microeng., vol. 18 , no. 9 , p. 095008 , Sep. 2008.

[37] B. López-Walle, M. Gauthier, and N. Chaillet, "Principle of a submerged freeze gripper for microassembly," IEEE Trans. Robot., vol. 24, no. 4, pp. 897-902, Aug. 2008.

[38] Y. Zhou and B. J. Nelson, "Adhesion force modeling and measurement for micromanipulation," in Proc. SPIE Conf. Microrobot. Micromanipulation, Boston, MA, Nov. 1998, pp. 169-180.

[39] F. Arai, D. Ando, and T. Fukuda, "Adhesion forces reduction for micro manipulation based on microphysics," in Proc. Int. Workshop Micro Electro Mech. Syst., San Diego, CA, Feb. 1996, pp. 354-359.

[40] R. A. Bowling, "A theoretical review of particle adhesion," in Particles on Surfaces I: Detection, Adhesion and Removal, K. L. Mittal, Ed. New York: Plenum, 1988, pp. 129-155.

[41] O. H. Pakarinen, A. S. Foster, M. Paajanen, T. Kalinainen, J. Katainen, I. Makkonen, J. Lahtinen, and R. M. Nieminen, "Towards an accurate description of the capillary force in nanoparticle-surface interactions," Model. Simul. Mater. Sci. Eng., vol. 13, no. 7, pp. 1175-1186, Oct. 2005.

[42] S. C. Chen and J. F. Lin, "Detailed modeling of the adhesion force between an AFM tip and a smooth flat surface under different humidity levels," J. Micromech. Microeng., vol. 18, no. 11, p. 115006 , Nov. 2008.

[43] J. N. Israelachvili, Intermolecular and Surface Forces, 2nd ed. New York: Academic, 1992

[44] A. Hariri, J. Zu, and R. B. Mrad, "Modeling of wet stiction in microelectromechanical systems (MEMS)," J. Microelectromech. Syst., vol. 16, no. 5, pp. 1276-1285, Oct. 2007.

[45] W. Ding, A. J. Howard, M. D. M. Peri, and C. Cetinkaya, "Rolling resistance moment of microspheres on surfaces: Contact measurements," Philos. Mag., vol. 87, no. 36, pp. 5685-5696, Dec. 2007.

[46] W. Ding, H. Zhang, and C. Cetinkaya, "Rolling resistance moment-based adhesion characterization of microspheres," J. Adhes., vol. 84, no. 12, pp. $996-1006$, Dec. 2008

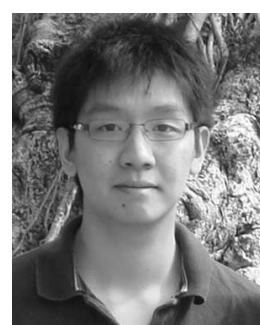

Brandon K. Chen (S'09) received the B.S. degree in mechanical engineering from Queen's University, Kingston, ON, Canada, in 2003. He is currently working toward the M.S. degree in the Department of Mechanical and Industrial Engineering, University of Toronto, Toronto, ON, Canada, where he is with the Advanced Micro and Nanosystems Laboratory.

His research interests include the design, fabrication, and testing of MEMS micro-nanogrippers and the use of these devices for micronanomanipulation.

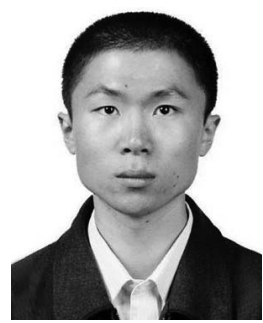

Yong Zhang (S'09) received the B.S. and M.S degrees in mechatronics engineering from Harbin Institute of Technology, Harbin, China, in 2005 and 2007 , respectively. He is currently working toward the Ph.D. degree in the Department of Electrical and Computer Engineering, University of Toronto, Toronto, ON, Canada, where he is with the Advanced Micro and Nanosystems Laboratory.

His research interests include the manipulation and characterization of micrometer- and nanometer-sized materials using MEMS and robotics approaches.

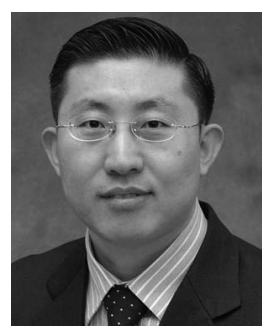

Yu Sun (S'01-M'03-SM'07) received the B.S degree in electrical engineering from Dalian University of Technology, Dalian, China, in 1996, the M.S. degree from the Institute of Automation, Chinese Academy of Sciences, Beijing, China, in 1999, and the M.S. degree in electrical engineering and the $\mathrm{Ph} . \mathrm{D}$. degree in mechanical engineering from the University of Minnesota, Minneapolis, in 2001 and 2003, respectively.

His research areas include the design and fabrication of M/NEMS devices, micro-nanorobotic manipulation, and the manipulation and characterization of biological cells, biomolecules, and nanomaterials. He is currently an Assistant Professor with the Department of Mechanical and Industrial Engineering and is jointly appointed in the Institute of Biomaterials and Biomedical Engineering and the Department of Electrical and Computer Engineering, University of Toronto, Toronto, ON, Canada. He held a Research Scientist position at the Swiss Federal Institute of Technology (ETH-Zürich), Zürich, Switzerland, before joining the faculty of the University of Toronto in July 2004. He is the Canada Research Chair in Micro and Nano Engineering Systems. 